\title{
APPLICATION OF B-SPLINE METHOD IN SURFACE FITTING PROBLEM
}

\author{
Fateme Esmaeili *, AliReza Amiri-Simkooei ${ }^{1}$, Vahab Nafisi ${ }^{2}$, Amin Alizadeh Naeini ${ }^{3}$ \\ ${ }^{1}$ Department of Geomatics Engineering, Faculty of Civil Engineering and Transportation, University of Isfahan amiri@eng.ui.ac.ir \\ ${ }^{2}$ Department of Geomatics Engineering, Faculty of Civil Engineering and Transportation, University of Isfahan nafisi@eng.ui.ac.ir \\ ${ }^{3}$ Department of Geomatics Engineering, Faculty of Civil Engineering and Transportation, University of Isfahan \\ a.alizadeh@eng.ui.ac.ir
}

KEY WORDS: Least-squares approximation, B-spline functions, surface fitting, splines' basis functions, 2D data analysis, continuity constrains

\begin{abstract}
:
Fitting a smooth surface on irregular data is a problem in many applications of data analysis. Spline polynomials in different orders have been used for interpolation and approximation in one or two-dimensional space in many researches. These polynomials can be made by different degrees and they have continuous derivative at the boundaries. The advantage of using B-spline basis functions for obtaining spline polynomials is that they impose the continuity constraints in an implicit form and, more importantly, their calculation is much simpler. In this study, we explain the theory of the least squares B-spline method in surface approximation. Furthermore, we present numerical examples to show the efficiency of the method in linear, quadratic and cubic forms and it's capability in modeling changes in numerical values. This capability can be used in different applications to represent any natural phenomenon which can't be experienced by humans directly. Lastly, the method's accuracy and reliability in different orders will be discussed.
\end{abstract}

\section{INTRODUCTION}

Spline functions play an important role in interpolation and approximation data analysis. Many researchers have used these functions and their advantages in their studies (Ahlberg, et al. 2016; Amiri-Simkooei, et al. 2018; Bartels, et al. 1995; Zahra and Van Daele 2018; Zangeneh-Nejad, et al. 2017). Selection of a suitable basis function to obtain the splines' polynomials is important in splines' computation. Among different spline basis functions, three kinds of them, namely, 1- truncated power splines, 2-cardinal splines and 3-B-splines have been attracted lots of attention. B-splines have usually been preferred because of their simple calculation and being well-conditioned, while cardinal splines are difficult to deal with and truncated power bases are open to ill-conditioned (De Boor 1972). To explain the properties of B-splines functions, we can mention their application in arbitrary data, having non-zero value only over intervals between the first knot and the last one, having a constant and distinct equation over the interval between two knots. Finally, imposition of the continuity constraints in functions and also their derivatives. Figure 1 presents a typical cubic B-spline.

(Hayes and Halliday 1974) presented a method which employs products of B-splines to represent the bicubic splines. The method can be extended to more dimensions and has already been used on problems in four independent variables. We will explain the theory and the application of least square B-spline method based on this research.

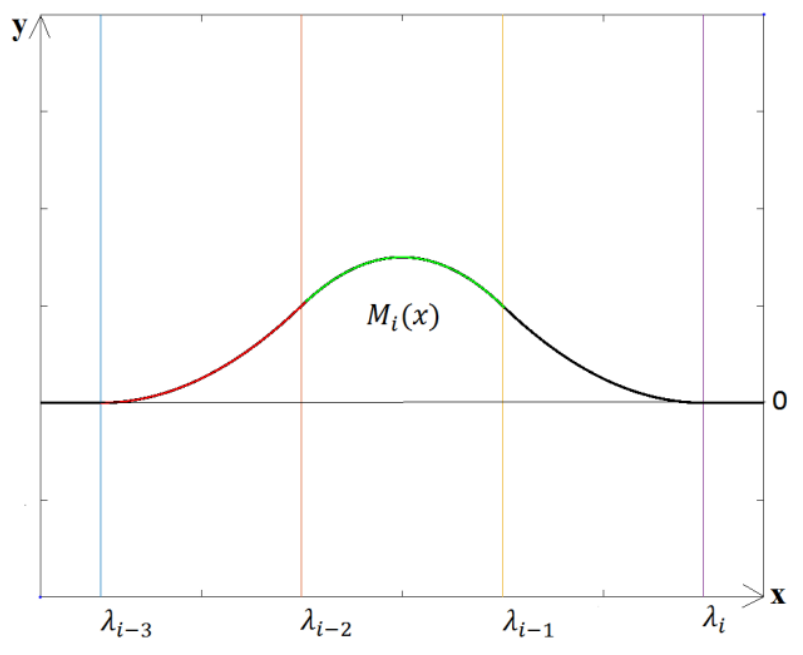

Figure 1. A typical B-spline with one variable, $\lambda_{i-3}$ and $\lambda_{i}$ stands for first and last knot, respectively.

In subsequent sections, we explain the theory of forming the Bspline functions in one variable in section 2 and then we expansion the theory in two variables in section3, the best solution for the problem will be fined in section4, section5 describes the equations in linear and quadratic forms, finally in section6 we present an example to evaluate the efficiency of the method, in the conclusion section we discuss about the probable applications of the method in different field.

\footnotetext{
* Department of Geomatics Engineering, Faculty of Civil engineering and transportation, University of Isfahan, Fatima.esmaeili74@gmail.com
} 


\section{FORMULATION IN ONE VARIABLE}

In order to produce a $\mathrm{B}$-spline over $\lambda_{i-3}<x<\lambda_{i}$ range, similar to figure 1 , we need to expand the knots out of boundary points:

$\int \lambda_{-3}<\lambda_{-2}<\lambda_{-1}<\lambda_{0} \leq a$

$\left\{\mathrm{b}<\lambda_{h+1}<\lambda_{h+2}<\lambda_{h+3}<\lambda_{h+4}\right.$

The numerical value of these knots can be chosen arbitrary and then, the number of fundamental B-spline functions is $h+4$ where $h$ stands for the number of knots in the problem's range.

$M_{i}(x), i=1,2, \ldots, h+4$

Discussed in (Curry and Schoenberg 1966), the typical cubic spline with knots $\lambda_{1}, \lambda_{2} \ldots . \lambda_{h}$ has a unique representation in the domain $a \leq x \leq b$ of the following form:

$S(x)=\sum_{i=1}^{h+4} \gamma_{i} \cdot M_{i}(x)$

Accordingly, the curve fitting problem can be considered as a least squares problem where the coefficients in equation (3) are estimated, from the following equations:

$\sum_{i=1}^{h+4} \gamma_{i}, M_{i}\left(x_{r}\right)=f_{r} \quad r=1,2, \ldots \ldots, m$

Where $f_{r}$ Stands for the observation vector, $M_{i}\left(x_{r}\right)$ stands for the fundamental B-splines, $\gamma_{i}$ stands for the unknown coefficients and $m$ shows the number of observations. This equation can be written in a matrix notation as:

$A \gamma=f$

In the equation (5), $A$ matrix has $m \times(h+4)$ dimensions, whose element in row $r$ of column $i$ is $M_{i}\left(x_{r}\right)$.

$\gamma, f$ Are column vectors with $\gamma_{i}, f_{r}$ elements, respectively. If the value of $x$ lies in the interval $\lambda_{j-1}<x<\lambda_{j}$, the corresponding row will have four nonzero value $M_{j}(x), M_{j+1}(x), M_{j+2}(x), M_{j+3}(x) \quad$ which are adjacent. If the data points are arranged in an increasing $x$ order. The special structure will be called band structure with the bandwidth four.

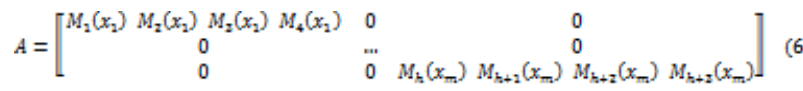

\section{FORMULATION IN TWO VARIABLES}

This is about surface fitting problem, we have to fit $f_{r}$ by using the least squares method in two independent variables $\left(y_{r}, x_{r}\right)$ in the $(x, y)$ plane. In order to gridding the plane we need to consider a rectangle in the $a \leq x \leq b, c \leq y \leq d$ domain which include all of the $\left(y_{r}, x_{r}\right)$ observations. We subdivide each of the ranges $[a, b],[c, d]$ by using knots.

$a=\lambda_{0}<\lambda_{1}<\ldots<\lambda_{h+1}=b$ $c=\mu_{0}<\mu_{1}<\cdots<\mu_{k+1}=d$

These knots partition the $R$ rectangle to $R_{i j}$ grids. Then the spline in the $R$ domain will be described by the following equation:

$\sum_{s=1}^{3} \sum_{t=1}^{3} a_{s, t} x^{s} y^{t}$

Equation (8) has continuity up to second partial derivatives. We need a set of fundamental splines just as we need in the one variable case, such set can be obtained by product of two onedimensional sets. One set of fundamental splines with respect to the $\lambda_{0}<\lambda_{1}<\cdots<\lambda_{h+1}$ in the $x$ range and one set of fundamental splines with respect to the $\mu_{0}<\mu_{1}<\cdots<\mu_{k+1}$ in the $y$ range. We can define a set of $N_{j}(y) \cdot M_{i}(x)$ fundamental spline in the two variables by product of two one-dimensional sets $M_{i}(x)$ and $N_{j}(y)$.

$\sum_{\mathrm{i}=1}^{\mathrm{h}+4} \sum_{j=1}^{k+4} \gamma_{\mathrm{i} j} M_{\mathrm{i}}\left(x_{r}\right) N_{j}\left(y_{r}\right)=f_{r} \quad r=1,2, \ldots \ldots m$

Where $\mathrm{m}=$ number of observations and $h+4$ stands for the number of fundamental splines $M_{i}(x)$, in x domain, and $k+4$ stands for the number of fundamental splines $N_{j}(x)$, in y domain.

Just like the one variable case, the knots expand out of the boundaries:

$\left\{\begin{array}{c}\lambda_{-3}<\lambda_{-2}<\lambda_{-1}<\lambda_{0}<a \\ \mathrm{~b}<\lambda_{h+1}<\lambda_{h+2}<\lambda_{h+3}<\lambda_{h+4}\end{array}\right.$
$\left\{\begin{array}{c}\mu_{-3}<\mu_{-2}<\mu_{-1}<\mu_{0}<c \\ \mathrm{~d}<\mu_{k+1}<\mu_{k+2}<\mu_{k+3}<\mu_{k+4}\end{array}\right.$

Equation (9) also has a matrix notation:

$A \gamma=f$

A Is a matrix with $(h+4) \times(k+4)$ columns and $m$ rows, whose elements has $N_{j}(y) \cdot M_{i}(x)$ value in row $r$ of $\operatorname{column}(j-1)(h+4)+i$.

The matrix $A$ takes the form below, if the data points are arranged in an increasing form of $(v=c t e) u=1,2, \ldots, h+1$ for $v=1,2, \ldots, k+1$.

$\left.A=\llbracket \begin{array}{cccc}A_{11} A_{12} A_{13} & A_{14} & 0 & 0 \\ 0 & \cdots & 0 \\ 0 & 0 & A_{k+1, k+1} & A_{k+1, k+2} \\ 0 & A_{k+1, k+3} & A_{k+1, k+4}\end{array}\right]$

Each of the $A_{r s}$ elements has band structure (bandwidth 4, as one variable case). 


\section{LEAST SQUARE SOLUTION}

There are many available solutions to estimate the $\gamma_{i j}$ unknown coefficients. In the least square B-spline method, we use least square solution to estimate these coefficients. The advantage of using this standard method is that, it minimizes $e^{T} Q_{y}^{-1} e=(y-A x)^{T} Q_{y}^{-1}(y-A x)$ where $\mathrm{A}$ is the design matrix, $Q_{y}$ is the covariance matrix of observable $y$, and e stands for the residuals vector. (Teunissen, et al. 2005). We obtain the coefficients by using equation (13) based on this method.

$\widehat{\gamma_{u}}=\left(A^{T} Q_{y}^{-1} A\right)^{-1} A^{T} Q_{y}^{-1} f$

Where $\gamma_{i j}=$ unknown coefficients, and $f$ is the observation vector.

\section{FORMULATION IN LINEAR AND QUADRATIC ORDERS}

As we mentioned before splines can be produced in any degrees, splines of order $\mathrm{p}$ usually have continuous derivatives up to order p-1 at the boundaries points (Amiri-Simkooei, et al. 2018). In the least square B-spline method, we need a set of Bspline functions for the construction of spline polynomials. It is obvious that splines of order $\mathrm{p}$ are constructed from B-splines of order $\mathrm{p}$, therefore, we need quadratic B-splines and linear Bsplines to fit quadratic spline surface and linear spline surface respectively, to our data. It should be noted that the linear Bsplines doesn't impose the continuity constraints of derivatives, due to first order equations.

The calculation of the least square B-spline method in linear and quadratic forms, is similar to the cubic one. The only difference is in the expansion of knots beyond the domain of problem. In the cubic case we needed 7 additional knots out of the boundaries (equation (10)). In quadratic and linear cases we need 5 and 3 additional knots respectively.

Quadratic form: $\left\{\begin{array}{c}\lambda_{-2}<\lambda_{-1}<\lambda_{0} \leq a \\ \mathbf{b}<\lambda_{h+1}<\lambda_{h+2}<\lambda_{h+3}\end{array}\right.$

Linear form: $\left\{\begin{array}{c}\lambda_{-1}<\lambda_{0} \leq a \\ \mathrm{~b}<\lambda_{h+1}<\lambda_{h+2}\end{array}\right.$

With respect to these knots, the form of the observation equations will be as followed:

Quadratic form:

$\sum_{i=1}^{h+3} \sum_{j=1}^{k+3} \gamma_{i j} M_{i}\left(x_{r}\right) N_{j}\left(y_{r}\right)=f_{r} \quad r=1,2, \ldots, m(16)$
Linear form:

$\sum_{i=1}^{h+2} \sum_{j=1}^{k+2} \gamma_{i j} M_{i}\left(x_{r}\right) N_{j}\left(y_{r}\right)=f_{r} \quad r=1,2, \ldots \ldots, m(17)$

In equations $(16,17), N_{j}\left(y_{r}\right), M_{i}\left(x_{r}\right)$ are basis B-spline functions in two variables.

\section{EXPERIMENTAL RESULTS}

We considered a mathematical function $f(x, y)=x e^{\left(-x^{2}-y^{2}\right)}$ and produced 500 random points on $-2 \leq x \leq 2$ and $-2 \leq y \leq 2$ domain. Figure 2 shows the location of these data points on the problem domain.

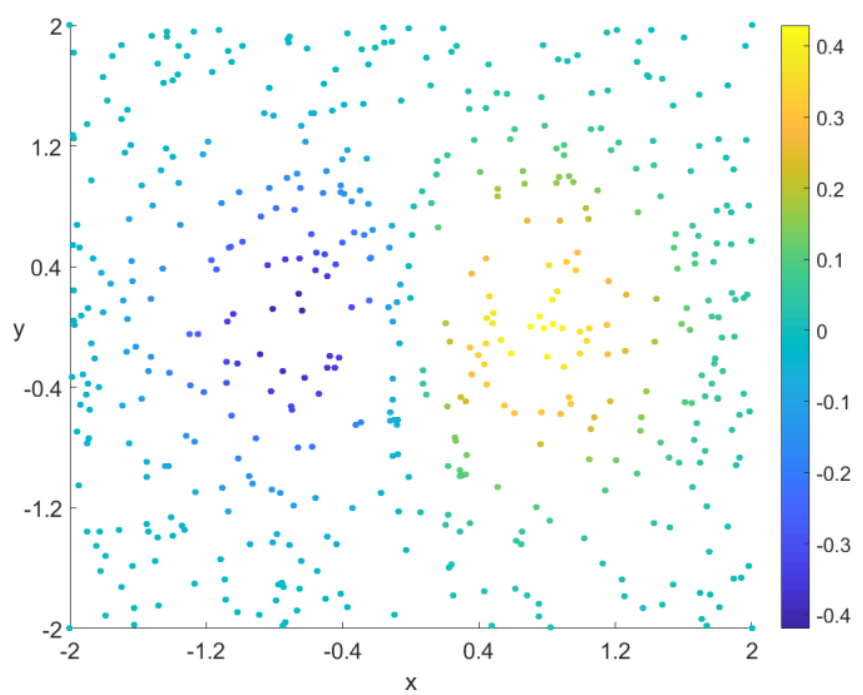

Figure 2. Location of data points over the whole domain

\subsection{NUMERICAL RESULTS}

We used least square B-spline method in linear, quadratic and cubic orders to fit a surface to our data, in each of the cases discussed in table 1 .

\begin{tabular}{|c|c|c|c|}
\hline method & $\begin{array}{c}\text { LS- } \\
\text { linear- } \\
\text { B-spline }\end{array}$ & $\begin{array}{c}\text { LS- } \\
\text { quadratic- } \\
\text { B-spline }\end{array}$ & $\begin{array}{c}\text { LS-cubic- } \\
\text { B-spline }\end{array}$ \\
\hline $\begin{array}{c}\text { number of } \\
\text { unknown } \\
\text { coefficients }\end{array}$ & 36 & 49 & 64 \\
\hline $\begin{array}{c}\text { number of } \\
\text { observations }\end{array}$ & 500 & 500 & 500 \\
\hline $\begin{array}{c}\text { number of } \\
\text { patches }\end{array}$ & 25 & 25 & 25 \\
\hline
\end{tabular}

Table 1.number of coefficients in different orders

As can be seen in this table, the number of unknown coefficients increases by increasing the order of B-splines. 
Figure 3 illustrates the orientation of B-spline gridding over the domain.

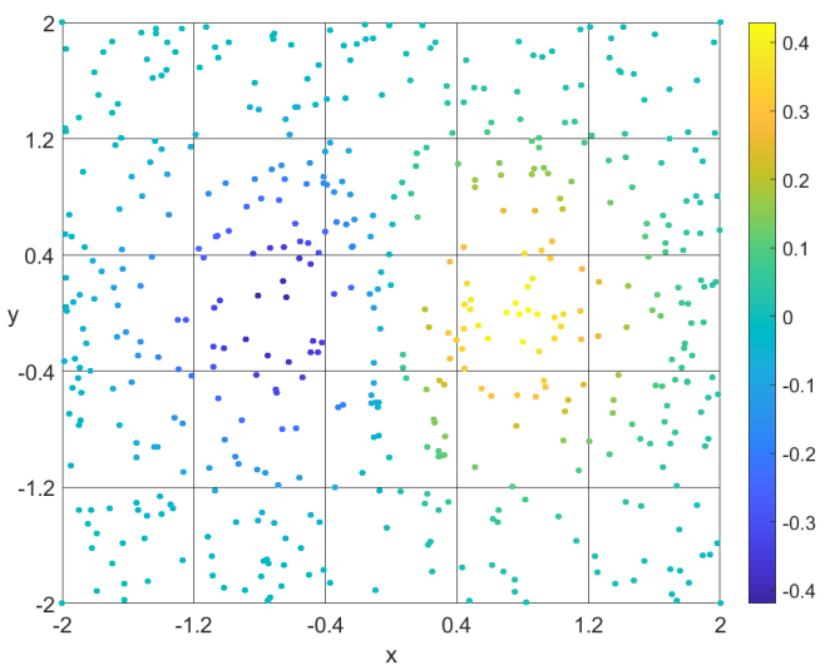

Figure 3. Orientation of B-splines' boundaries over the domain

We evaluate application of each method, mentioned in table 1 , by numerical value of mean residual. The numerical value of the residual in each of the data points, is calculated from the following equation.

$R=\left|\hat{f}_{r}-f_{r}\right|$

Where $\widehat{f}_{r}$ and $f_{r}$ are estimated value and original value for $r$ th point, respectively.

In each of the cases, we calculated mean value of $R$. We considered this value as an assessment criterion to judge about the effect of B-splines' order in the accuracy of results (Table 2).

\begin{tabular}{|c|c|c|c|}
\hline method & $\begin{array}{c}\text { LS-linear- } \\
\text { B-spline }\end{array}$ & $\begin{array}{c}\text { LS-quadratic- } \\
\text { B-spline }\end{array}$ & $\begin{array}{c}\text { LS- } \\
\text { cubic-B- } \\
\text { spline }\end{array}$ \\
\hline $\begin{array}{c}\text { numerical } \\
\text { value of mean } \\
\text { residuals }\end{array}$ & 0.014 & 0.0112 & 0.0027 \\
\hline
\end{tabular}

Table 2. Mean residual in different orders

As can be found in this table, the numerical value of mean residual in the least square cubic B-spline is the least value comparing with other orders. This value increases by decreasing the order of B-splines.

\subsection{VISUAL RESULTS}

The least square B-spline method has ability to show the results in a continuous form. This exclusive property causes the method to be useful in modeling $2 \mathrm{~d}$ data. We fitted a surface on our data, and then represented our results in a $3 \mathrm{~d}$ continuous form. By comparing the models obtained from different methods, we can demonstrate how good B-splines can approximate the original function (Figure 4).

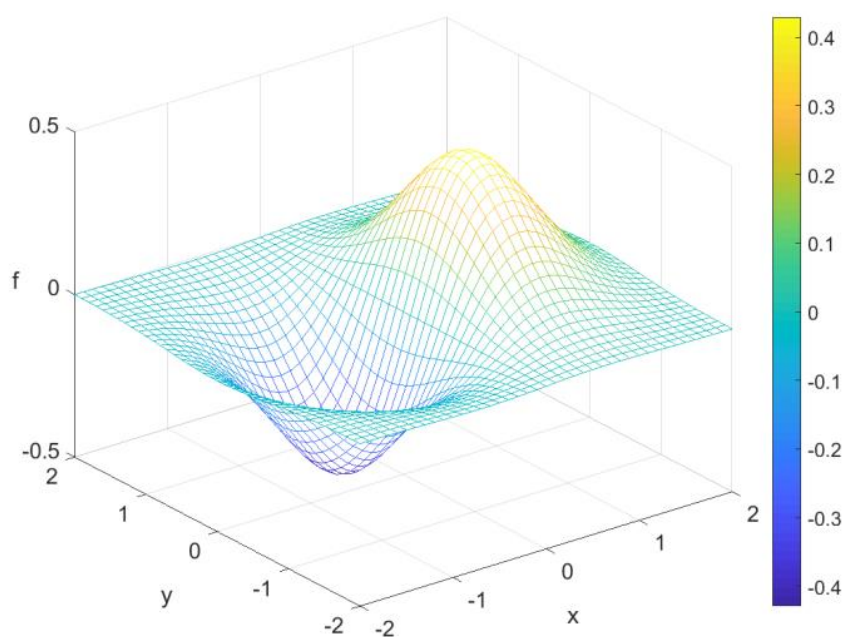

Figure 4. Original function

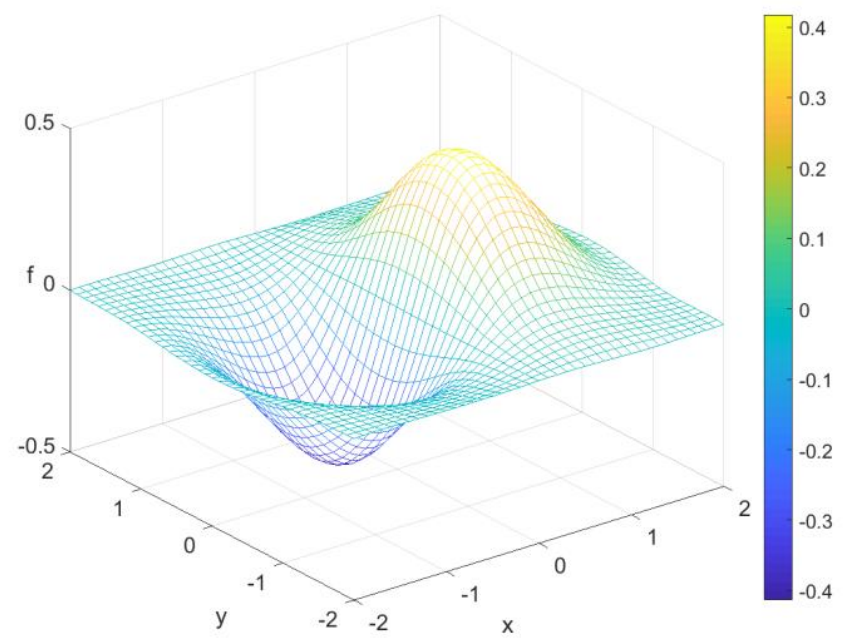

Figure 5. Fitted surface obtained from cubic least squares

B-spline 


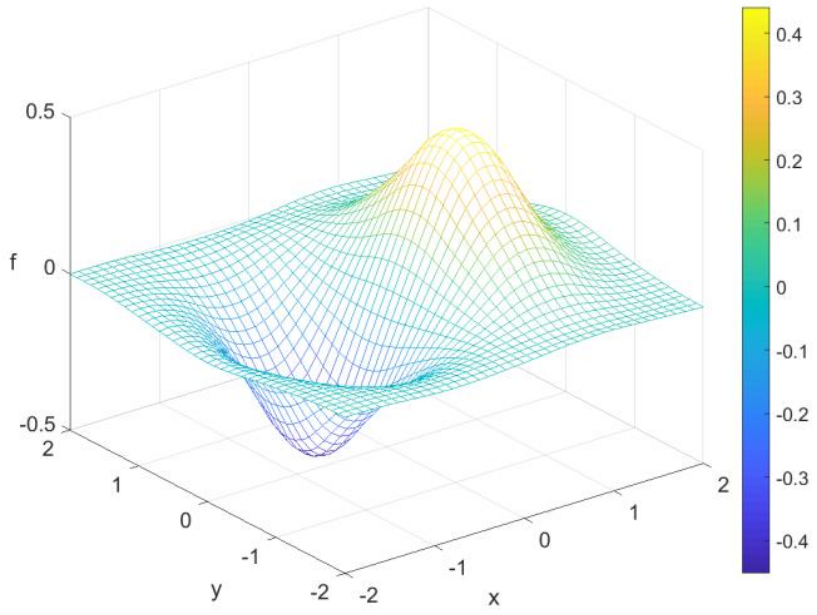

Figure 6. Fitted surface obtained from quadratic least squares Bspline

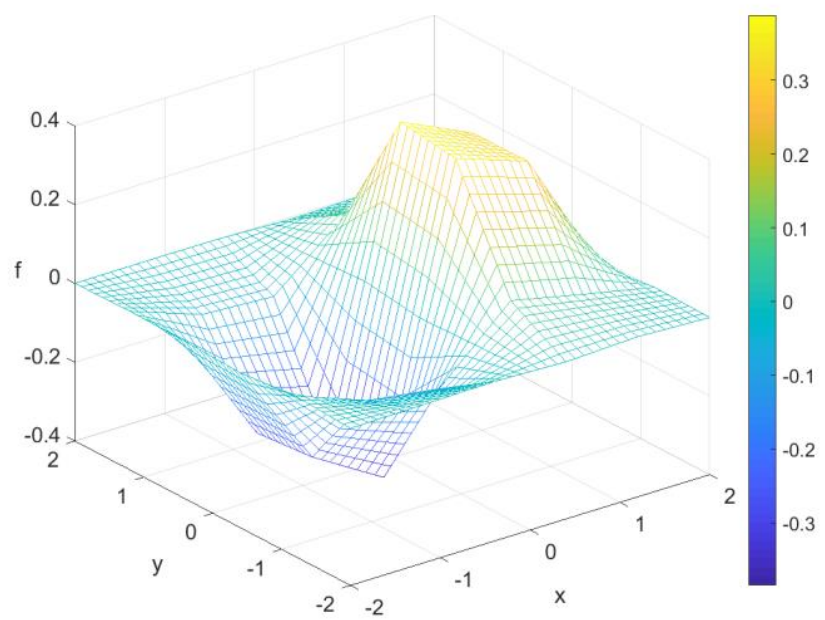

Figure 7. Fitted surface obtained from linear least squares

$$
\text { B-spline }
$$

As can be seen in figures $(5,6,7)$, the least square B-spline method acted efficiently in representing the changes in original function. The continuous behaviour of the function is clearer in the models obtained from quadratic and cubic orders. Next following figures show the numerical value of residuals over the problem domain, in different orders.

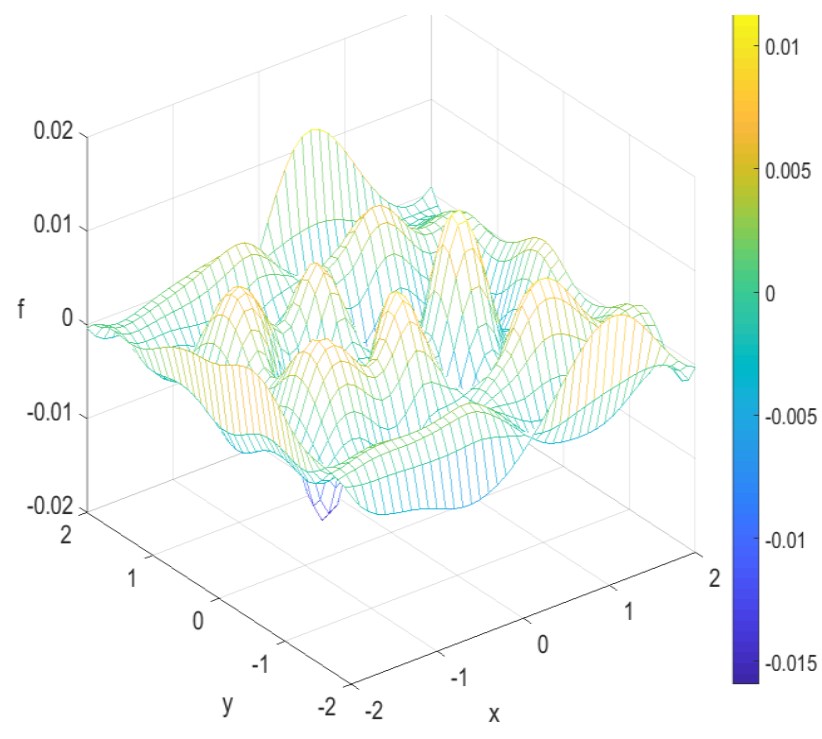

Figure 8. Error in fitting cubic least squares B-spline surface

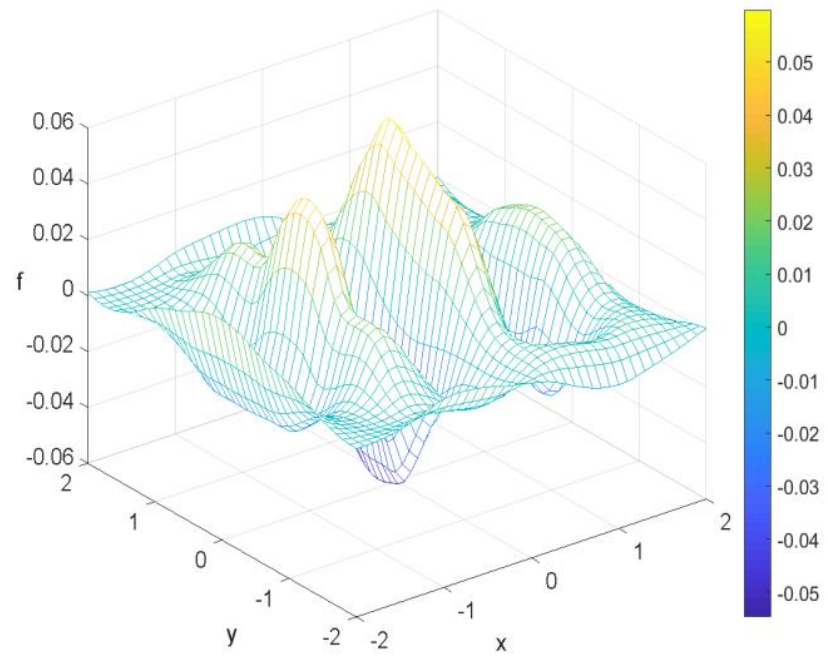

Figure 9. Error in fitting quadratic least squares B-spline surface

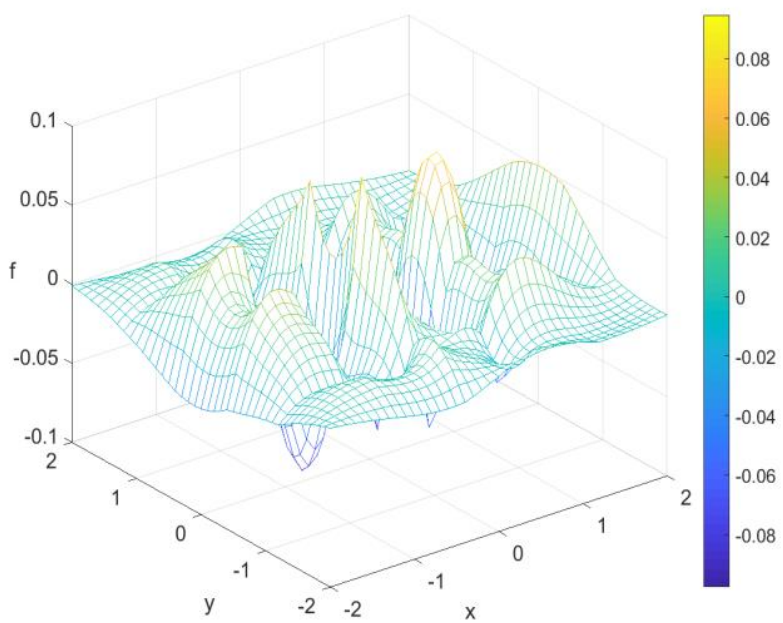

Figure 10. Error in fitting linear least squares B-spline surface 
By comparing these figures we can find out that range of errors in linear order is 5 times bigger than the cubic order. The boundaries' errors happened more in the linear order than the other ones.

\section{CONCLUTION}

The least square B-spline method can be considered as one of the powerful methods in data analysis. The method performs efficiently in providing accurate numerical results and representing the behavior of data over the problem domain. This capability make the method useful in modeling natural phenomenon which may have sudden changes on their behavior. The visual results of the method are applicable in representing the location of unexpected behavior on data. The cubic and quadratic orders of this method are preferred in dealing with continuous spaces. The linear order of this method can have some inefficiency in boundaries' area. The accuracy of the method increases by increasing the order of B-splines.

\section{REFERENCES}

Ahlberg, J. H., E. N. Nilson and J. L. Walsh (2016). The Theory of Splines and Their Applications: Mathematics in Science and Engineering: A Series of Monographs and Textbooks, Elsevier.

Amiri-Simkooei, A., M. Hosseini-Asl and A. Safari (2018). "Least squares 2D bi-cubic spline approximation: Theory and applications." $\underline{\text { Measurement. }}$

Curry, H. B. and I. J. Schoenberg (1966). "On Pólya frequency functions IV: the fundamental spline functions and their limits." Journal d'analyse mathématique 17(1): 71-107.

De Boor, C. (1972). "On calculating with B-splines." Journal of Approximation theory 6(1): 50-62.

Hayes, J. G. and J. Halliday (1974). "The least-squares fitting of cubic spline surfaces to general data sets." IMA Journal of Applied Mathematics 14(1): 89-103.

Teunissen, P., D. Simons and C. Tiberius (2005). "Probability and observation theory. Delft Institute of Earth Observation and Space Systems (DEOS)." Delft University of Technology, The Netherlands.

Zahra, W. and M. Van Daele (2018). "Discrete Spline Solution of Singularly Perturbed Problem with Two Small Parameters on a Shishkin-Type Mesh." Computational Mathematics and Modeling: 1-15.

Zangeneh-Nejad, F., A. Amiri-Simkooei, M. Sharifi and J. Asgari (2017). "Cycle slip detection and repair of undifferenced single-frequency GPS carrier phase observations." $\underline{\text { GPS }}$ Solutions 21(4): 1593-1603. 